\title{
A model for codon position bias in RNA editing
}

\author{
Tsunglin Liu and Ralf Bundschuh \\ Department of Physics, Ohio State University, 191 W Woodruff Av., Columbus OH 43210-1117
}

\begin{abstract}
RNA editing can be crucial for the expression of genetic information via inserting, deleting, or substituting a few nucleotides at specific positions in an RNA sequence. Within coding regions in an RNA sequence, editing usually occurs with a certain bias in choosing the positions of the editing sites. In the mitochondrial genes of Physarum polycephalum, many more editing events have been observed at the third codon position than at the first and second, while in some plant mitochondria the second codon position dominates. Here we propose an evolutionary model that explains this bias as the basis of selection at the protein level. The model predicts a distribution of the three positions rather close to the experimental observation in Physarum. This suggests that the codon position bias in Physarum is mainly a consequence of selection at the protein level.
\end{abstract}

PACS numbers: 87.23.Kg, 05.40.-a, 87.14.Gg, 87.14.Ee

\section{INTRODUCTION}

The central dogma of molecular biology states that the transfer of genetic information flows from the genomic DNA to messenger RNA to proteins. This implies that there are two fundamental processes involved in the fabrication of proteins. The first such process is transcription and it consists of copying the genomic DNA into a messenger RNA of identical sequence. Both, the genomic DNA and the messenger RNA use an alphabet of four letters which makes direct copying of one sequence into the other possible. The second process is called translation and consists of synthesizing a protein, i.e., a sequence of amino acids, following the instructions contained in the sequence of the messenger RNA. During translation groups of three consecutive bases of the messenger RNA - the codons - are read in order to determine which of the 20 amino acids is to be appended to the protein being synthesized. The map from the $4^{3}=64$ possible codons into the 20 possible amino acids is called the genetic code.

Before translation into proteins, it has been discovered that RNAs transcribed from most eukaryotic genes undergo a variety of processing events, that convert RNA precursors into mature RNAs ready for translation. For example, the splicing process removes extended stretches of the nucleotide sequences called introns from an RNA precursor such that only the remaining RNA sequence codes for a protein.

Besides these normal processing events, many novel phenomena that change the content of RNA sequences before translation have been discovered in several different organisms 1, 2, 3, 4, 5, 6, 7, 8, 9, 10, 11]. These phenomena, which are now coined as RNA editing, consist of inserting, deleting, or changing individual or a very small number of nucleotides. Still, even by changing only a few nucleotides RNA editing can significantly alter the coding and result in functionally distinct proteins. In addition, RNA editing has also been found to occur in non-coding regions like tRNAs and rRNAs, and can alter the function of these RNA molecules as well.
For editing within the coding regions, there is often a significant codon position bias. For example, it has been discovered that in the mitochondrial genes of the slime mold Physarum polycephalum, about two thirds of the editing events that insert Cs happen at the third positions of their respective codons $[\underline{5}, 6]$. On the contrary, in mitochondrial genes of Arabidopsis and other plants, about 90 percent of the editing events, which in this case convert Cs to Us, happen at the first two positions of the codons 12, 13, 14].

Codon position bias is somewhat surprising since there is no obvious relation between the editing which happens on the RNA level and codons which in principle have a meaning only during translation. The underlying reason why the editing machinery might prefer certain codon positions for editing is still not understood.

Here, we propose an evolutionary model which explains the editing position bias in mRNAs. In the evolutionary scheme, a population of an organism undergoes mutations which alter the DNA sequences of the individuals in the population and change their genetic information. Under natural selection, the members in the gene pool that carry the genes with higher fitness grow faster and increase their relative frequency in the total population.

The fitness of a gene in general depends on the encoded protein sequence as well as other biological parameters. In our model we will assume that the only dominant selection mechanism is the fitness of the resulting protein sequence. This assumption is reasonable if editing is relatively inexpensive, because in this case most editing sites will be random in nature rather than involve some other biological factors. Comparing our results with the actual codon position bias data then reveals if this assumption is true or if there are other, more fundamental selection mechanisms at work in a given organism.

The two cases we apply our model to are the mitochondrial mRNAs of Physarum polycephalum and of the plants Arabidopsis thaliana, Brassica napus, and Oryza sativa. Abundant editing events have been observed in these organisms. In Physarum mitochondrial mRNAs, 
one in every 25 bases is edited on average, which leads to about 1 in every 8 codons being edited on average. In plant mitochondrial mRNAs, about $2 \%$ of the nucleotides are edited on average. In the remainder of this article, we briefly describe the editing events in these organisms. Then we focus first on the evolutionary model for Physarum. Following similar approaches for the case of Physarum, we then move on to the case of plant mitochondria.

Mitochondrial RNAs of Physarum polycephalum have been found to be edited extensively by insertions of mono-(C,U) and dinucleotides (CU,GU,UA,AA,GC, and UU) [5, 6]. Among the editing events within mRNAs of Physarum polycephalum, C insertions are the most frequent events. In plant mitochondria, the most abundant events are $\mathrm{C}$ to $\mathrm{U}$ conversions. Thus, we only focus on these most frequent editing events in this communication.

For C insertions in 11 mitochondrial mRNAs of Physarum polycephalum [15], some editing positions are ambiguous as nucleotide Cs are inserted right next to another C. Excluding these ambiguous editing sites, $227 \mathrm{C}$ insertions are observed within the coding regions. Among these, 58 and 24 insertions occur at the first and second positions of the codons, respectively. The remaining 145 insertions are found at the third codon position. In this case, the third position is the most favorable and the second position is the least favorable as already noted in previous work comprising only 5 mitochondrial $\mathrm{mR}$ NAs [16].

In plant mitochondria, the dominant editing event is a $\mathrm{C}$ to $\mathrm{U}$ conversion. Thus, there is no ambiguity in determining the codon position of these events. The editing codon position preferences of the three plant mitochondria studied here are quite similar. In Arabidopsis, 154/236/51 C to U conversions have been observed at the three positions 12]. In Oryza 13] and Brassica 14], $142 / 243 / 33$ and $174 / 230 / 77$ conversions have been observed respectively. This suggests that the editing position bias stems from the same mechanism for the three organisms. Notice that the order of the position bias is the opposite to that of Physarum.

We now develop our model for the insertional editing events of Physarum. This model will later be easily adapted to the case of substitutional editing such as in the plant mitochondria.

The scheme of our evolutionary model is the following. During the proliferation of Physarum, nucleotide mutations occur at random positions in the mitochondrial DNA sequence. These mutations can be substitutions, insertions, and deletions. Although we model all three types of mutations, we are mostly interested in deletions in the DNA sequence. Most offspring with a deletion die because the protein produced according to the mutated DNA sequence is out of frame from the site of the deletion on and thus cannot function properly. However, some few deletions may be accepted by the edit-

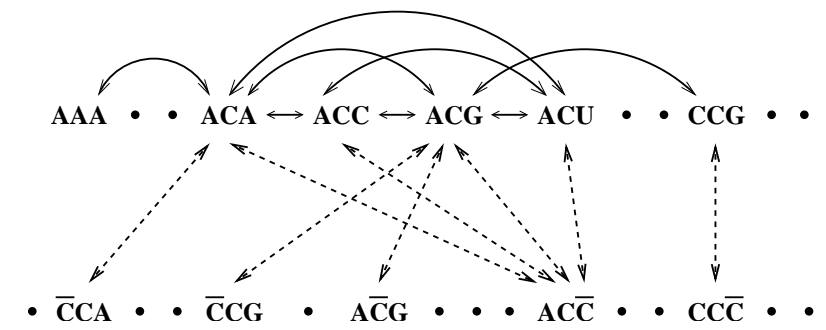

FIG. 1: Mutations among codons of the evolutionary model for Physarum. Solid lines stand for mutations between regular codons. Dashed lines are for mutations between a regular codon and a codon with an editing site.

ing machinery which would insert back nucleotides $\mathrm{C}$ to those positions of mutation and thus rescue them. Under natural selection, these offspring would survive if the resulting protein could function properly in place of the original one.

The genetic code, i.e., the rules of translation from codons to amino acids, is organized such that often the third codon position is irrelevant to the identity of the amino acid interpreted into the protein sequence. Thus, the third codon position is the least sensitive to nucleotide changes to $\mathrm{C}$ generated by editing events. Therefore, in this mutation-selection model, random deletions at the third codon position survive much better than at the first and second position.

Beyond this qualitative picture, the random mutations and selection can be rigorously formulated in the following way using as an example the codon ACG. For random mutations, we assume the substitutions and deletions occur randomly at one of the three positions with certain mutation rates $\mu_{s}$ and $\mu_{e}$. The random substitutions result in 9 regular codons by converting the base in each position into 3 other nucleotides (Fig. 1). The random deletions generate 3 codons with editing sites $\bar{C} C G$, or $\mathrm{A} \bar{C} \mathrm{G}$, or $\mathrm{AC} \bar{C}$, where the bar stands for a vacant site in the DNA sequence after mutation. As an amino acid is expressed, this vacant site will be treated as a $\mathrm{C}$ to represent the editing event in which a nucleotide $\mathrm{C}$ is inserted before translation. Note that $\mu_{e}$ is the effective rate for a deletion that is accepted by the editing machinery and rescued by inserting a $\mathrm{C}$. This rate is in general smaller than the base deletion rate. For codons with a vacant site, they also undergo insertions which randomly insert one of the four nucleotides $(\mathrm{A}, \mathrm{C}, \mathrm{G}, \mathrm{U})$ back into the vacant site with a mutation rate $\mu_{e}$, which we take to be the same as the random deletion rate.

The basic assumption of our model is that selection happens only at the amino acid level. Thus, the fitness of a codon, i.e., the growth rate, depends only on the similarity of the new amino acid coded for by the mutated codon to the original amino acid. To be specific, we will use the amino acid scoring matrix BLOSUM62 [17] to quantify the similarities between amino acids. However, 
our results are not very sensitive to the choice of this matrix. As an example, the initial codon ACG is assigned a large growth rate of 6 because it translates into the desired amino acid threonine. The mutated codon $\bar{C} \mathrm{CG}$ would express the amino acid proline and is assigned a growth rate of -1 since proline behaves quite differently from thrieonine according to the BLOSUM62 matrix.

Apart from the fitness at the amino acids level, a codon created by an editing event is considered as less fit than a regular one because the editing machinery presumably uses up resources in order to perform the editing. We thus reduce the growth rate for a codon created by an editing event by an editing cost $c$.

In order to cast this model in mathematical terms we define the fraction of codon $i$ as $x_{i}$, where $i=1 \sim 64$ is for the 64 regular codons and $i=65 \sim 112$ is for all codons containing an editing site. The corresponding growth rates and editing costs are defined as $g_{i}$ and $c_{i}$, where $g_{i}$ is obtained from the BLOSUM62 matrix and $c_{i}=c$ for $i=65 \sim 112$ and zero otherwise. We then write the general model as

$$
\begin{aligned}
\dot{x_{i}} & =\left(g_{i}-c_{i}-\sum_{j \neq i} \mu_{i j}\right) x_{i}+\sum_{j \neq i} \mu_{j i} x_{j}-x_{i}\left(\sum_{j} g_{j} x_{j}\right) \\
& =\sum_{j} M_{i j} x_{j}-x_{i}\left(\sum_{j} g_{j} x_{j}\right)
\end{aligned}
$$

where $\mu_{i j}$ is the general mutation rate from codon $i$ to codon $j$, and is set as $\mu_{s}$ for substitutions, $\mu_{e}$ for deletions and insertions, and 0 for codons that cannot be mutated into each other.

We will look at the distribution of regular codons and codons with editing sites in this model at equilibrium. Although the last term in this equation is non-linear, Eigen's theory [18, 19] tells us that the equilibrium distribution is proportional to the eigenvector of the matrix $M_{i j}$ for the largest eigenvalue. Thus, obtaining the equilibrium distribution is merely a matter of numerically finding the eigenvector for the largest eigenvalue of a $112 \times 112$ matrix which we do with Mathematica [20].

To obtain some insight into this evolutionary model, we again first look at the case where the initial codon is ACG. We will focus on the limit of strong selection in which most results become largely independent of the individual mutation rates $\mu_{s}$ and $\mu_{e}$. We obtain this strong selection limit by setting $\mu_{s}$ and $\mu_{e}$ to be much smaller than 1 since the growth rates that set the time scale are of order 1 . To be more specific, we set $\mu_{s}=\mu_{e}=$ $10^{-6}$ because the relative magnitude of $\mu_{s}$ to $\mu_{e}$ only slightly affects the result. As expected, the equilibrium distribution shows that only those codons that translate into the original amino acid survive. Thus, among the codons with editing sites, only $\mathrm{A} \bar{C} \mathrm{~A}, \mathrm{~A} \bar{C} \mathrm{C}, \mathrm{A} \bar{C} \mathrm{G}, \mathrm{A} \bar{C} \mathrm{U}$, $\mathrm{AC} \bar{C}$ survive since the genetic code depends only on the first two positions in this case. We also find that the ratio between editing at the second position and at the third position is about one. Thus, we arrive at a simple scheme in the strong selection limit that only the mutations that result in the same amino acid survive and each position that survives contributes equally at equilibrium.

This simple scheme allows us to quickly predict the ratio among edited positions for each codon. The overall ratio is then easily obtained by considering all possible codons with proper weights. The weights of the codons are determined by the experimentally observed unedited codon frequencies in the DNA sequence. This scheme results in the ratio of editing positions as $17 / 11 / 72$ percent which is rather close to the experimental observed ratio 25/11/64 percent.

The above result is obtained without any editing cost, i.e., $c=0$. We find that the role of the editing cost is mainly to reduce the fraction of the total number of codons with editing sites; the ratio among the editing positions is only slightly changed upon introducing an editing cost. Thus, by adjusting the editing cost, we can tune the fraction of codons with $\mathrm{C}$ insertions to match the experimental result of about $7 \%$. In this case, the overall ratio of editing positions is about $19 / 15 / 66$ percent, which is still very close to the experimental result.

We conclude that this simple evolutionary model provides a possible scenario for the editing codon position bias. This suggests that in Physarum the majority of editing events might indeed be subject to no other biological constraint but the fitness of the resulting protein sequence. Thus, editing events might be randomly acquired.

This is also consistent with the evolution of RNA editing in Physarum and its close relatives [21]. Abundant editing has also been observed in the organisms Didymium nigripes and Stemonitis flavogenita. However, in Arcyria cinerea and Clastoderma debaryanum, only few $\mathrm{C}$ insertions or even none are observed. This indicates that the editing machinery in Arcyria and Clastoderma is not yet fully developed. The fact that Physarum has acquired so many editing sites since the divergence from Arcyria and Clastoderma suggests that editing in Physarum is less expensive and that thus random editing is likely.

To study plant mitochondrial genes, we slightly modify our evolutionary model as follows. The random substitutions between regular codons remain the same as in the case of Physarum, and they occur with a rate $\mu_{s}$. As to the editing events that convert Cs to Us, we assume that for certain bases that mutate to $\mathrm{Cs}$, the editing machinery would recognize these sites and convert them to Us before translation. Here, we use $\bar{U}$ to represent such a mutation. Thus, as an example, the codon ACG can mutate into $\bar{U} \mathrm{CG}, \mathrm{A} \bar{U} \mathrm{G}$, and $\mathrm{AC} \bar{U}$ as the corresponding nucleotide mutate to a $\mathrm{C}$ and then is recognized by the editing machinery. This process involving an editing 
event occurs with a mutation rate $\mu_{e}$. Again, the back mutation that converts a $\bar{U}$ to one of the four nucleotides $(\mathrm{A}, \mathrm{C}, \mathrm{G}, \mathrm{U})$ is modeled to also occur with the same mutation rate $\mu_{e}$. Mathematically, the evolutionary model for Physarum and plant mitochondria are identical.

This evolutionary model, when applied to plant mitochondrial genes, does not predict the correct preference in the codon positions for editing. Instead, it still predicts the majority of editing sites to occur at the third codon position. This suggests that in plant mitochondrial genes editing events do not just happen randomly, but that there exist some other biological mechanisms that bias the choice of editing sites.

This assertion is consistent with what is known about the editing mechanism in subcellular organelles of plants. Recent experiments show that different editing sites in subcellular organelles of plants usually require different proteins in the editing process [22]. In such a case, each editing site is quite expensive in terms of resources the cell has to provide. It is highly unlikely that such expensive editing sites are randomly acquired. The existence of editing events in spite of the fact that they are expensive is an indication that these editing sites are significantly involved in biological functions beyond simply providing the correct protein sequence.

In summary, the closeness of the result of our evolutionary model to the experimental observations in Physarum suggests that the editing position bias in Physarum is mainly a consequence of random mutations with selection at the protein level. In the case of plant mitochondria, the disagreement between the evolutionary model and the observations implies that some other biological factors besides selection at the protein level must also play a role in the evolution, so that the random mutation-selection scheme does not fit in these organisms.

We gratefully acknowledge contributions by Taeyoung
Choi to the very early stages of this project and fruitful discussions with Jonatha Gott. This work has been supported by grant no. DMR-0404615 from the National Science Foundation.

[1] H. Grosjean, R. Benne, eds. 1998. Modification and Editing of RNA, Washington, DC: ASM Press.

[2] B. Bass, ed. 2000. RNA editing: Frontiers in Molecular Biology. Oxford Univ. Press

[3] R. Benne et al., Cell 46, 819 (1986)

[4] L. Simpson and J. Shaw, Cell 57, 355 (1989)

[5] R. Mahendran, M. R. Spottswood and D. L. Miller, Nature 349, 434 (1991)

[6] J. M. Gott, L. M. Visomirski, and J. L. Hunter, J. Biol. Chem. 268, 25483 (1993)

[7] J. M. Gualberto et al., Nature 341, 660 (1989)

[8] P. S. Covello and M. W. Gray, Nature 341, 662 (1989)

[9] R. Hiesel, B. Wissinger, W. Schuster, and A. Brennicke, Science 246, 1632 (1989)

[10] L. M. Powell et al., Cell 50, 831 (1987)

[11] S. H. Chen et al., Science 238, 363 (1987)

[12] P. Giegé and A. Brennicke, PNAS 96, 15324 (1999)

[13] H. Handa, Nucl. Acids. Res., 31, 20 (2003)

[14] Y. Notsu et al., Mol. Genet. Genomics 268, 434 (2002)

[15] N. Parimi, R. Bundschuh and J. M. Gott (to be published)

[16] D. Miller et al., Sem. Cell Biol. 4, 261 (1993)

[17] S. Henikoff and J. G. Henikoff, PNAS.89, 10915 (1992)

[18] J. B. Plotkin, J. Dushoff, M. M. Desai, H. B. Fraser, q-bio.PE/0410013 (2004)

[19] C. J. Thompson and J. L. McBride, Math. Biosci. 21, 127 (1973)

[20] Wolfram Research, Inc., Mathematica, Version 5.0, Champaign, IL (2003).

[21] T. L. Horton and L .F. Landweber, RNA 6, 1339 (2000)

[22] T. Miyamoto, J. Obokata, M. Sugiura, Mol. Cell Biol. 22, 6726 (2002) 